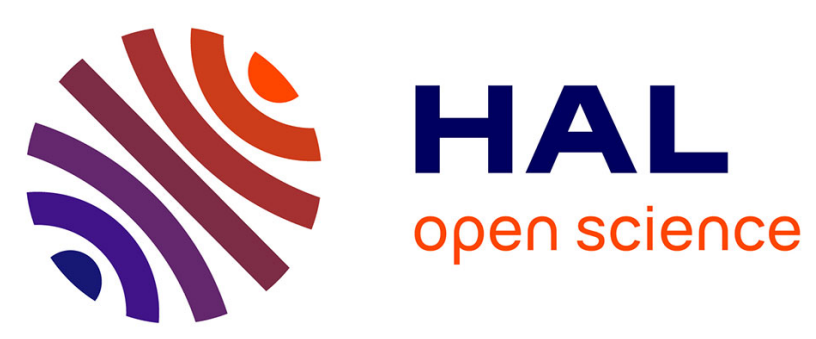

\title{
Negative ion characterization in a helicon plasma source for fusion neutral beams by cavity ring-down spectroscopy and Langmuir probe laser photodetachment
}

R Agnello, Stéphane Bechu, I Furno, P H Guittienne, A A Howling, R Jacquier, G Plyushchev, M Barbisan, R Pasqualotto, I Morgal, et al.

\section{To cite this version:}

R Agnello, Stéphane Bechu, I Furno, P H Guittienne, A A Howling, et al.. Negative ion characterization in a helicon plasma source for fusion neutral beams by cavity ring-down spectroscopy and Langmuir probe laser photodetachment. Nuclear Fusion, 2020, 60, pp.026007. 10.1088/1741-4326/ab5e64 . hal-03049113

\section{HAL Id: hal-03049113 \\ https://hal.science/hal-03049113}

Submitted on 9 Dec 2020

HAL is a multi-disciplinary open access archive for the deposit and dissemination of scientific research documents, whether they are published or not. The documents may come from teaching and research institutions in France or abroad, or from public or private research centers.
L'archive ouverte pluridisciplinaire HAL, est destinée au dépôt et à la diffusion de documents scientifiques de niveau recherche, publiés ou non, émanant des établissements d'enseignement et de recherche français ou étrangers, des laboratoires publics ou privés. 


\section{Negative ion characterization in a helicon plasma source for fusion neutral beams by Cavity Ring-Down Spectroscopy and Langmuir Probe laser photodetachment}

To cite this article before publication: Riccardo Agnello et al 2019 Nucl. Fusion in press https://doi.org/10.1088/1741-4326/ab5e64

\section{Manuscript version: Accepted Manuscript}

Accepted Manuscript is "the version of the article accepted for publication including all changes made as a result of the peer review process, and which may also include the addition to the article by IOP Publishing of a header, an article ID, a cover sheet and/or an 'Accepted

Manuscript' watermark, but excluding any other editing, typesetting or other changes made by IOP Publishing and/or its licensors"

This Accepted Manuscript is () EURATOM 2019.

During the embargo period (the 12 month period from the publication of the Version of Record of this article), the Accepted Manuscript is fully protected by copyright and cannot be reused or reposted elsewhere.

As the Version of Record of this article is going to be / has been published on a subscription basis, this Accepted Manuscript is available for reuse under a CC BY-NC-ND 3.0 licence after the 12 month embargo period.

After the embargo period, everyone is permitted to use copy and redistribute this article for non-commercial purposes only, provided that they adhere to all the terms of the licence https://creativecommons.org/licences/by-nc-nd/3.0

Although reasonable endeavours have been taken to obtain all necessary permissions from third parties to include their copyrighted content within this article, their full citation and copyright line may not be present in this Accepted Manuscript version. Before using any content from this article, please refer to the Version of Record on IOPscience once published for full citation and copyright details, as permissions will likely be required. All third party content is fully copyright protected, unless specifically stated otherwise in the figure caption in the Version of Record.

View the article online for updates and enhancements. 
Negative ion characterization in a helicon plasma source for fusion neutral beams by Cavity Ring-Down Spectroscopy and Langmuir Probe laser photodetachment

\author{
R Agnello', S Béchu ${ }^{2}$, I Furno ${ }^{1}$, Ph Guittienne ${ }^{3}$, A A Howling ${ }^{1}$, \\ R Jacquier ${ }^{1}$, G Plyushchev ${ }^{1}$, M Barbisan ${ }^{4,5}$, R Pasqualotto $^{4}$, I \\ Morgal $^{6}$, A Simonin 6 \\ ${ }^{1}$ Ecole Polytechnique Fédérale de Lausanne (EPFL), Swiss Plasma Center (SPC), \\ CH-1015 Lausanne, Switzerland \\ ${ }^{2}$ Université Grenoble-Alpes, CNRS, Grenoble INP, LPSC-INP23, 38000 Grenoble, \\ France \\ ${ }^{3}$ Helyssen, Route de la Louche 31, CH-1092 Belmont-sur-Lausanne, Switzerland \\ ${ }^{4}$ Consorzio RFX, Corso Stati Uniti 4, I-35127 Padova, Italy \\ ${ }^{5}$ INFN-LNL, Viale dell'Università 2, I-35020 Legnaro, Italy \\ ${ }^{6}$ CEA, IRFM, F-13108 St-Paul-lez-Durance, France \\ E-mail: riccardo.agnello@epfl.ch
}

Abstract.

Negative ions are characterized in the helicon plasma source RAID (Resonant Antenna Ion Device) at the Swiss Plasma Center by means of Cavity Ring-Down Spectroscopy (CRDS) and Langmuir Probe (LP)-assisted laser photodetachment. A high density and axially homogeneous plasma column is produced via a RF antenna able to sustain the propagation of helicon waves in a steady state regime. An electron density $n_{e} \cong 2.0 \times 10^{18} \mathrm{~m}^{-3}$ in $\mathrm{H}_{2}$ plasma at $0.3 \mathrm{~Pa}$ and $3 \mathrm{~kW}$ of input power is measured in the center of the plasma column by LP and microwave interferometry. The electron temperature profile is peaked on axis reaching $T_{e} \approx 5 \mathrm{eV}$ and decreasing to $1.5 \mathrm{eV}$ at $r=0.05 \mathrm{~m}$. Thus, a hot core region forms where $\mathrm{H}_{2}$ molecules are rovibrationally excited $\left(\mathrm{H}_{2}(\nu)\right)$, and a cold edge, where low energy electrons can attach to $\mathrm{H}_{2}(\nu)$ and produce $\mathrm{H}^{-}$ions by dissociative attachment. In this work we use LP-assisted laser photodetachment and CRDS diagnostics to measure $\mathrm{H}^{-}$and $\mathrm{D}^{-}$radial density profiles and how they depend on source parameters. We show that negative ions are distributed on a shell of $0.06 \mathrm{~m}$ radius with a peak value of $\sim 2.0 \times 10^{16} \mathrm{~m}^{-3}$ in $\mathrm{H}_{2}$ plasma. These results suggest that, although substantial technical development is needed, helicon plasmas could be considered as a possible candidate as sources of negative ions for future NBIs. 
Negative ion characterization in a helicon plasma source

\section{Introduction}

Negative ion based Neutral Beam Injectors (NBIs) for fusion applications require high accelerated negative ion currents, and current density, with the ion energy of the order of $1 \mathrm{MeV}$. In conventional NBI systems, positive ions, usually $\mathrm{H}^{+}$or $\mathrm{D}^{+}$, are accelerated by electric fields and neutralized by charge exchange with gas molecules. The neutralization efficiency, however, drops to negligible values for energies greater than $250 \mathrm{keV}$, rendering gas neutralization impracticable for positive ions [1]. The neutralization efficiency of negative $\mathrm{H}^{-}$or $\mathrm{D}^{-}$ions in gas neutralizers, is around $60 \%$ at the energy requirements of NBIs, rendering negative ions much more attractive. For example, ITER's negative ion-based NBI will deliver $16.7 \mathrm{MW}$ of $\mathrm{D}^{0}$ at $1 \mathrm{MeV}[2]$. The negative ions for ITER will be produced by Inductively-Coupled Plasma (ICP) sources requiring several hundreds of $\mathrm{kW}$ of $\mathrm{RF}$ power and the evaporation of caesium to enhance the negative ion production by surface processes [3]. For the next generation of NBI fusion devices, less power consumption will be essential to increase the overall efficiency of the power plant. This is crucial considering that DEMO NBI design will be driven by Reliability, Availability, Maintainability, Inspectability (RAMI) and high efficiency requirements [4]. In this context, helicon plasma sources could be considered to serve as sources of negative ions for NBIs for a/number of reasons. First, helicon plasma sources require less RF power compared to ICP sources to reach similar plasma density parameters [5]. Second, they can operate more easily than ICPs at pressures $<0.3 \mathrm{~Pa}$, which is essential in order to reduce to acceptable levels the negative ion losses due to electron stripping in the extractor and accelerator in a future NBI system. Third, negative ions are efficiently produced in volume [6], although it remains to be demonstrated that the yield is sufficiently high to avoid the use of Cs. Although helicon plasma sources have been extensively studied for their potential application for space thrusters [7], their application in fusion remains largely unexplored to date.

The implementation of a helicon-based negative ion source requires the investigation of negative ion physics including both experimental and theoretical efforts, as well as ensuring that the source will be compatible with an extractor and an acceleration system. Research performed in the Resonant Antenna Ion Device (RAID) [8] at the Swiss Plasma Center aims at broadening the current knowledge of the physics of volume production of negative hydrogen and deuterium ions in helicon plasmas, which has been explored, apart from RAID, to the best of our knowledge, only in a few helicon plasma devices $[9,10]$. The possible integration of such a source, if able to reach the RAMI and efficiency requirements compatible for future NBIs, requires considerable experimental, numerical and technical development. At this early stage of development, one relevant physics goal, which can be addressed on RAID, is to understand how the volume negative ion production yield depends on helicon plasma parameters such as the RF power, the neutral pressure and the magnetic field geometry.

RAID can sustain steady state plasma discharges in different gases, including $\mathrm{H}_{2}$, $\mathrm{D}_{2}$, using a resonant antenna in a birdcage geometry [11]. In RAID, many 


\section{Negative ion characterization in a helicon plasma source}

basic plasma diagnostics including Langmuir Probes (LPs), microwave interferometry, magnetic probes, Optical Emission Spectroscopy (OES) and diagnostics based on laser-plasma interaction, such as LP-assisted laser photodetachment and Cavity RingDown Spectroscopy (CRDS), are routinely employed $[12,13]$. Previous experimental campaigns based on OES and CRDS revealed a significant amount of negative ions both in hydrogen and deuterium plasmas $[6,14]$. Both OES and CRDS measure negative ion density in the order of a few $10^{16} \mathrm{~m}^{-3}$, and development is needed to reach the negative ion densities required for NBIs, which is $10^{17} \mathrm{~m}^{-3}[15]$. The main advantage of CRDS is that it allows a direct measurement of the absolute value of the negative ion density without requiring any calibration and being non-invasive. However, the line-integrated measurement does not provide the local density. In a previous experimental campaign, we tested the robustness of CRDS and studied the experimental challenge of how to reduce undesired effects that could deteriorate the accuracy of the measurement [14]. In this paper, we extensively exploit CRDS by varying plasma parameters in combination with LP-assisted laser photodetachment to deduce absolute negative ion density local profiles. The knowledge of these profiles is important since they are used as input parameters for negative ion extraction and modeling.

The paper is organized as follows: Section 2 describes the plasma source RAID. In Section 3, we present the experimental setup of CRDS and LP-assisted laser photodetachment on the RAID device. Section 4 shows the experimental results in $\mathrm{H}_{2}$ and $\mathrm{D}_{2}$ by varying the plasma parameters, and Section 5 discusses the conclusions and future perspectives.

\section{The Resonant Antenna Ion Device (RAID)}

RAID, shown in Fig. 1, produces a magnetized plasma column by means of a resonant antenna in a birdcage geometry [8]. The cylindrical vacuum vessel is surrounded by six coils producing an axial magnetic field up to $800 \mathrm{G}$ on axis. The first coil on the left is usually operated in inverse polarity with respect to the other five to create a gradient of magnetic field along the axis in the antenna region. This enhances the electron confinement in the antenna probably because slower electrons produced in the antenna region are confined by the bottleneck of the magnetic field. This was experimentally seen to facilitate the ignition of the plasma discharge and to stabilize the helicon regime. A variety of axial boundary conditions can be implemented. In the present experiments, the plasma column is bounded behind the antenna by a ceramic foam, and on the far end by a water-cooled grounded copper end-plate. A stable helicon regime can be attained with a minimum power of $1000 \mathrm{~W}$ for hydrogen and deuterium. A traveling helicon wave has been measured by means of a magnetic probe [13] confirming that the plasma column is sustained by helicon waves. 
Negative ion characterization in a helicon plasma source

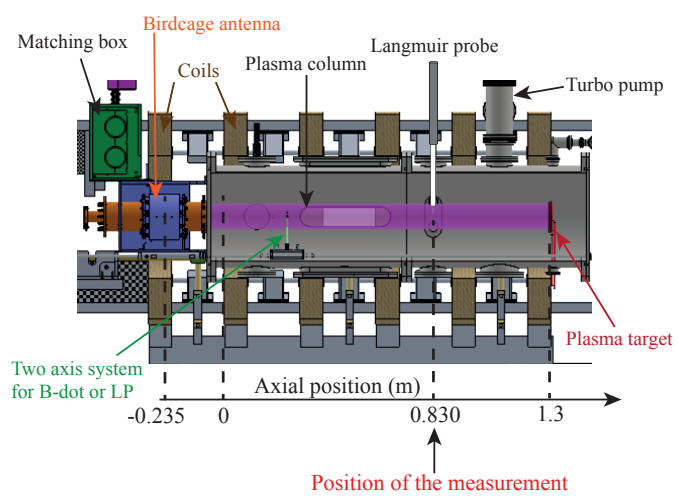

Figure 1. A cross section of the RAID plasma device along the axis. The cylindrical vacuum vessel of $1.4 \mathrm{~m}$ length and $0.4 \mathrm{~m}$ diameter is surrounded by six coils producing an axial magnetic field up to $800 \mathrm{G}$. The resonant birdcage antenna, placed on the left, produces a plasma column up to the target on the right.

\subsection{Electron density and temperature profiles}

Electron density and temperature along the vessel are measured by means of a movable LP on a two-axis system. Electrons are magnetized, with a Larmor radius which is comparable to, or even smaller than, the LP radius, making the analysis of IV curves to extract the absolute value of $n_{e}$ unreliable. Ions, however, are not magnetized. We estimate $\sim 0.1 \mathrm{eV}$ for the ion temperature by assuming approximate thermal equilibrium with the neutral gas whose temperature $\sim 1000 \mathrm{~K}$ is obtained from spectroscopic measurements [6]. In a magnetic field of $200 \mathrm{G}$, this gives an ion Larmor radius of $\sim 1.6 \mathrm{~mm}$, much bigger than the LP tip radius $(0.2 \mathrm{~mm})$. Absolute electron density profiles are therefore obtained with a LP in combination with a $100 \mathrm{GHz}$ microwave heterodyne interferometer or by Thomson Scattering diagnostic, currently in the commissioning phase. In the rest of the manuscript, we will refer to "fill pressure" for the gas pressure measured in the vacuum vessel prior to operation of the helicon device. In hydrogen, a typical peak electron density of $2.0 \times 10^{18} \mathrm{~m}^{-3}$ with $3 \mathrm{~kW}$ of injected power at $0.3 \mathrm{~Pa}$ fill pressure is usually achieved as depicted in Fig. 2, showing the electron density measured along the plasma vessel. The origin of the radial profile corresponds to the axis of the vacuum vessel.

The electron density along the axis of the plasma is quite uniform $( \pm 5 \%)$ in the region downstream of the antenna in the axial position between $0.4 \mathrm{~m}$ to $1.1 \mathrm{~m}$.

Fig. 3(a) shows the electron density radial profile, measured at the axial position $0.830 \mathrm{~m}$, which strongly increases with RF power, as expected by power balance considerations [16]. The helicon wave power is deposited along the plasma column and, in steady state equilibrium, results in radially peaked $n_{e}$ profiles. The $T_{e}$ profiles shown in Fig. 3(b) are, however, only weakly dependent on the RF power. This can be explained with simple particle balance considerations: at this plasma regime, the electron temperature is independent of the plasma density and therefore the input power, 
Negative ion characterization in a helicon plasma source

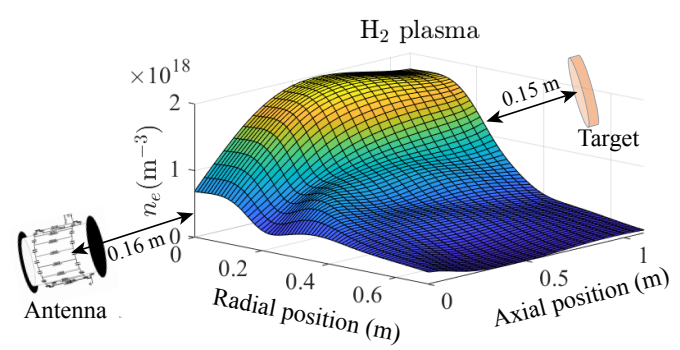

Figure 2. 3D cylindrically-symmetric interpolated profile of plasma density in the vessel measured with a two axis LP and calibrated with a $100 \mathrm{GHz}$ heterodyne microwave interferometer. Measurements start $0.16 \mathrm{~m}$ from the near end of the antenna and stop $0.15 \mathrm{~m}$ before the target.

(a) $3 \times 10^{1}$

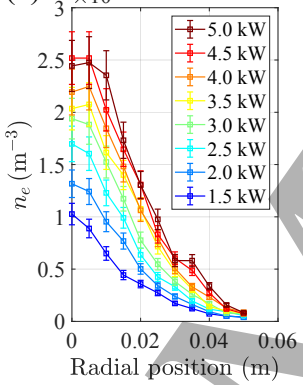

on $(\mathrm{m})$ (b) 6

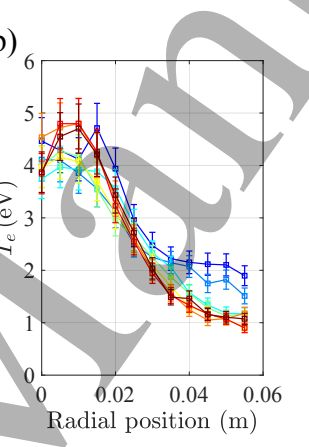

Figure 3. (a) Radial electron density and (b) temperature profile for a $\mathrm{H}_{2}$ plasma at $0.3 \mathrm{~Pa}$ fill pressure and $200 \mathrm{G}$ magnetic field on axis for different RF powers, at the axial position $0.830 \mathrm{~m}$. The density profile $n_{e}$ is obtained from LP measurements calibrated with interferometry. The $T_{e}$ profiles are weakly dependent on the RF power.

depending only on the gas pressure [16]. Similar behaviours are observed in other helicon sources [17]. The $n_{e}$ and $T_{e}$ profiles are measured with a L-shaped Langmuir probe (0.2 $\mathrm{mm}$ diameter and $10 \mathrm{~mm}$ long) located at axial position $0.830 \mathrm{~m}$ and vertically movable. The peaked $T_{e}$ profile is favorable for the volume production of $\mathrm{H}^{-}$since it provides a central hot region to rovibrationally excite the $\mathrm{H}_{2}$ molecules and a cold external region where low energy electrons can produce the $\mathrm{H}^{-}$by dissociative attachment (DA) $[18,19]$ :

$$
\mathrm{H}_{2}(v \geq 5)+e(\leq 1 \mathrm{eV}) \rightarrow \mathrm{H}+\mathrm{H}^{-} \quad(\mathrm{DA})
$$

There are two possible mechanisms to produce the rovibrationally excited $\mathrm{H}_{2}(v)$ in volume. The first is the stepwise vibrational excitation of molecules in collision with electrons (e-V process):

$$
e+\mathrm{H}_{2}(v) \rightarrow \mathrm{H}_{2}^{-} \rightarrow e+\mathrm{H}_{2}(v+\Delta v) \quad(\mathrm{e}-\mathrm{V})
$$

this reaction, however, requires many collisions to increase the vibrational state to high values since the most probable change in $v$ is $\Delta v= \pm 1$ [20]: At the low pressure typical 


\section{Negative ion characterization in a helicon plasma source}

of ions sources for NBIs, another important contribution to the production of the excited $\mathrm{H}_{2}(v)$ is the radiative decay from singlet state, the so called E-V process [21]:

$$
\begin{aligned}
& \mathrm{H}_{2}\left(X^{1} \Sigma_{g}^{+}, v\right)+e \rightarrow \mathrm{H}_{2}\left(B^{1} \Sigma_{u} ; C^{1} \Pi_{u}\right)+e \\
& \rightarrow \mathrm{H}_{2}\left(X^{1} \Sigma_{g}^{+}, v^{\prime \prime}\right)+e+h \nu .
\end{aligned}
$$

Previous experiments based on Optical Emission Spectroscopy (OES) and CRDS revealed a significant volume production of $\mathrm{H}^{-}$and $\mathrm{D}^{-}$[6]. The radial distribution measured by OES suggests that negative ions reach a maximum density in a radial shell located around $r \sim 4 \mathrm{~cm}$. Since CRDS was demonstrated to be a robust diagnostic, it is used here for an experimental campaign to broaden knowledge of negative ion physics in the present experimental device and to form a database for models of negative ion production in the helicon plasma. In order to make measurements comparable with the previous campaign [14], the same experimental setup was used as described in 3, combined with a movable LP to measure the radial profile of negative ion density using laser photodetachment.

\section{Experimental setup: laser-based diagnostics for negative ions}

To obtain absolute $\mathrm{H}^{-}$and $\mathrm{D}^{-}$radial density profiles, the measurements of CRDS and LP-assisted laser photodetachment were combined. CRDS has a fixed measurement position and is used to calibrate the relative radial profiles obtained by the LP-assisted laser photodetachment. The optical setups for the CRDS and for the LP-assisted photodetachement are respectively shown in Fig.4(a) and in Fig.4(b). In Fig.4(a), the measurement position of the laser is fixed at $r=0.04 \mathrm{~m}$ (where we expect the largest density of $\mathrm{H}^{-}$according to OES measurements [6]) and the laser beam performs multiple reflections between the two high reflectivity (HR) mirrors.

The CRDS technique was developed in 1988 by A. O'Keefe [22] to perform optical absorption measurements. CRDS is nowadays routinely used in fusion research as a plasma diagnostic to measure $\mathrm{H}^{-}$and $\mathrm{D}^{-}$line densities in negative ion sources $[15,23]$. To detect these ions, a pulsed Nd:YAG laser is employed [24]. The photon energy $(E=1.2 \mathrm{eV}$, corresponding to a wavelength of $\lambda=1064 \mathrm{~nm})$ is sufficient to strip the weakly bound electron of $\mathrm{H}^{-}$and $\mathrm{D}^{-}\left(E_{\text {binding }}=0.75 \mathrm{eV}\right)$ by photodetachment:

$$
h \nu+\mathrm{H}^{-} \rightarrow \mathrm{H}+\mathrm{e} .
$$

The CRDS diagnostic on RAID relies on an optical cavity made of two HR mirrors $(R>99.998 \%$ [24]) to multiply the interaction length with the absorbing medium. The light stored in the cavity is lost with an exponential decay time $\tau$ depending on the mirrors' reflectivity and the medium's absorption. Further details on the CRDS diagnostic in RAID can be found in Ref. [14]. An example of a CRDS signal in RAID is shown in the inset in Fig. 5 . The line-integrated negative ion density $\bar{n}_{-}$is:

$$
\bar{n}_{-}=\int n_{-}(l) d l
$$


1

2

3

4

5

6

7

8

9

\section{Negative ion characterization in a helicon plasma source}

where $n_{-}$is the local negative ion density. The line-averaged negative ion density $\left\langle n_{-}\right\rangle$ is given by:

$$
\left\langle n_{-}\right\rangle=\frac{1}{\sigma_{-}} \frac{L}{c d}\left(\frac{1}{\tau}-\frac{1}{\tau_{0}}\right),
$$

where $\tau$ and $\tau_{0}$ are the decay times in the presence and absence of absorbing medium $\left(\mathrm{H}^{-}\right.$or $\left.\mathrm{D}^{-}\right) . L$ is the cavity length, $\sigma_{-}$the photodetachment cross section, $c$ the speed of light and $d$ the distance over which the density is averaged. Thus, the line-integrated negative ion density is $\bar{n}_{-}=d \cdot\left\langle n_{-}\right\rangle$The reflectivity of the mirrors is a key parameter

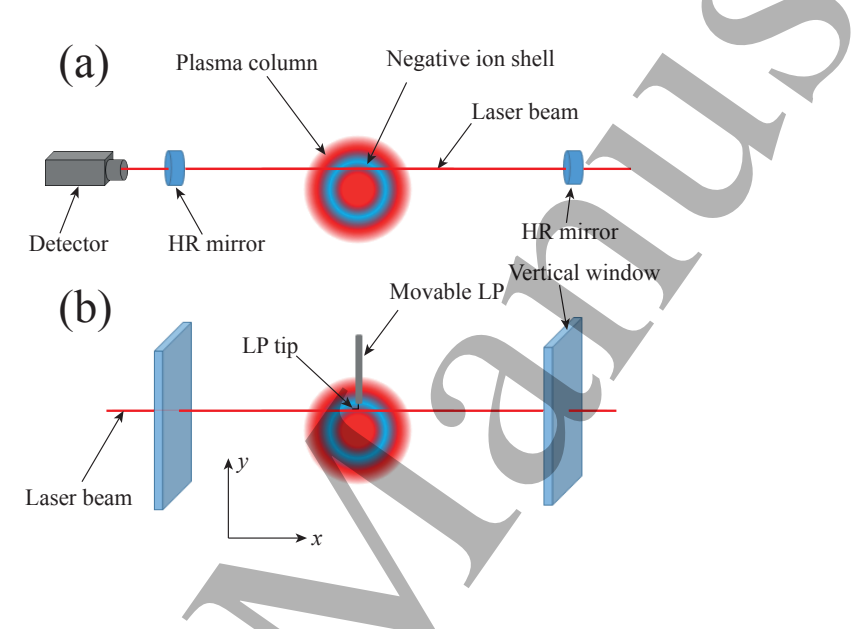

Figure 4. A schematic of the two different experimental setups employed: in (a) the CRDS and, in (b) the LP-laser photodetachment scheme. In (a) the measurements position of the laser is fixed at $4 \mathrm{~cm}$. In (b) the laser beam is vertically displaced simultaneously with the LP.

since it determines the signal-to-noise ratio and hence the minimum detectable negative ion density.

CRDS at a fixed measurement position is not sufficient to determine the local negative ion density since the CRDS signal is determined by the interaction between the photons in the laser beam and the plasma all along the path of the laser beam in the plasma. Moving the laser beam across the plasma column would allow to Abel invert the data to reconstruct the $n$-radial profile. However, this method is impracticable due to the lengthy alignment procedure. LP-assisted laser photodetachment can be used to obtain relative negative ion profiles with a spatial precision comparable to the laser beam diameter. The movable LP employed on RAID for the LP-assisted laser photodetachment is shown schematically in Fig. 4(b). The laser measurement position can be varied simultaneosly with the movable LP, which can be vertically displaced. The probe is displaced by a stepper motor and the vacuum is maintained by a bellow. The probe tip is aligned co-axially with the direction of the laser beam and biased at $V_{\text {bias }}=26 \mathrm{~V}$, above the plasma potential $V_{\text {plasma }}$, which is $V_{\text {plasma }}=12( \pm 1) \mathrm{V}$ for $r<0.06 \mathrm{~m}$, for both $\mathrm{H}_{2}$ and $\mathrm{D}_{2}$ plasmas, and independent of gas pressure. The $\mathrm{H}^{-}$or D- ions in the laser path are photoneutralized and the stripped electrons are collected by the probe. This causes a sudden increase of the signal detected by the LP, in the 


\section{Negative ion characterization in a helicon plasma source}

order of a fraction of microsecond, proportional to the local negative ion density. An example of a photodetachment signal in RAID in shown in Fig. 7. The fast transient is measured by a current probe consisting of a toroidal current transformer. The local negative ion density $n_{-}$can be estimated as follows:

$$
\frac{n_{-}}{n_{e}}=\frac{I_{p d}}{I_{d c}}
$$

where $n_{e}$ is the local electron density, $I_{d c}$ the collected current at the $V_{\text {bias }}$, and $I_{p d}$ the amplitude of the variation of the collected current due to the photodetachment. Eq. (6) can be applied if the following criteria are satisfied [25]: 1) the laser wavelength is suitable to photo-detach the electrons of the negative ions (i.e. the photon energy and the detachment cross section are sufficiently high), 2) the laser pulse energy is enough to photodetach all negative ions in the target volume, 3 ) the laser beam must have a minimum diameter from which the amplitude of the signal does not increase with beam diameter and, 4) the probe bias must be above the plasma potential. These criteria are satisfied as described above and in section 4.2 .

\section{Experimental Results}

The CRDS and the LP-assisted photodetachment were used in two distinct experimental campaigns in $\mathrm{H}_{2}$ and $\mathrm{D}_{2}$ plasmas with $200 \mathrm{G}$ magnetic field on the axis of RAID, and variable gas pressures.

\subsection{CRDS characterization in RF power and gas pressure at a fixed Line-Of-Sight} $(L O S)$

CRDS measurements are performed employing the technique described below, which was already introduced in [14] and reviewed here for clarity. Fig. 5 shows $\tau$, the ringdown time, as a function of time, before, during, and after a $\mathrm{H}_{2}$ plasma discharge with $5 \mathrm{~kW}$ RF power. The laser is pulsed at $10 \mathrm{~Hz}$ so that the temporal resolution is of the order of $0.1 \mathrm{~s}$. The plasma discharge starts at $t \cong 30 \mathrm{~s}$ in the presence of a mixture of $\mathrm{H}_{2}$ and Ar gas (see Eig. 5); the Ar gas is added to facilitate plasma ignition [14]. The drop of $\tau$ just after plasma ignition is due to a combination of the absorption of the laser pulse by negative ions and the thermal stress of the optical cavity. Just after plasma ignition, Ar is removed in a few seconds and RF power is increased meanwhile. In the plateau where $\tau \cong 37 \mu \mathrm{s}$, the plasma consists purely of $\mathrm{H}_{2}$ and it is steady state. The plasma discharge is then turned off at $t \cong 80 \mathrm{~s}$ causing the sudden increase of $\tau$. To determine the line-integrated negative ion density $\bar{n}_{-}, \tau$ is linearly fitted $10 \mathrm{~s}$ before and after the $\tau$ sudden increase, so that accurate values of $\tau$ just before and after the jump can be estimated. The $\tau$ after the plasma shut down does not recover immediately to the pre-discharge value since the optical cavity was deformed by thermal stress during the plasma discharge. After a few tens of seconds, the complete recovery to the pre-discharge value is observed. 
1

2

3

4

5

6

7

8

9
Negative ion characterization in a helicon plasma source

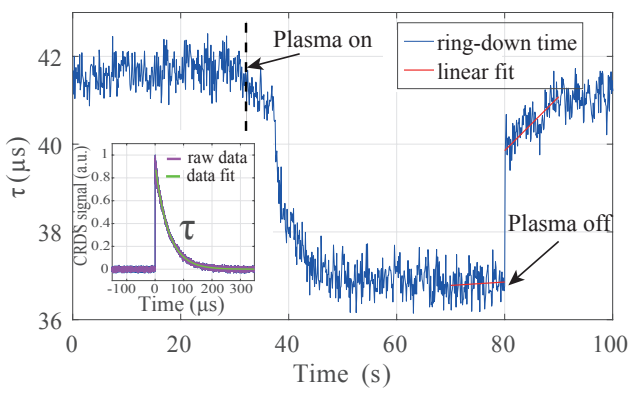

Figure 5. Ring-down time $\tau$ before, during, and after a plasma discharge for a $\mathrm{H}_{2}$ plasma and $5 \mathrm{~kW}$ RF power. The plasma discharge starts at $t \cong 30 \mathrm{~s}$ and it is stabilized after a few seconds. Plasma is turned off at $t=80 \mathrm{~s}$ causing the jump of $\tau$ due to the disappearance of $\mathrm{H}^{-}$ions. The linear fit of tau before and after the plasma off is used to accurately determine $\tau$ at plasma off. The jump is used to evaluate the line-averaged $\mathrm{H}^{-}$density. The inset shows a fit as used to calculate the ring-down time for each data point $\tau$.

Thus, the accuracy of the measurement relies on the determination of the $\tau$ jump, whose detectability in turn depends on the mirrors' reflectivity and optical cavity's stability. Once the reliability of the technique was established [14], power scans were performed. Fig. 6 shows measurements with $\mathrm{H}_{2}$ and $\mathrm{D}_{2}$ plasma for different RF powers and gas pressures. $\mathrm{H}^{-}$and $\mathrm{D}^{-}$line-integrated density increase with $\mathrm{RF}$ power for both plasmas. For power larger than $4 \mathrm{~kW}$, however, the growth rate is reduced for $\mathrm{H}^{-}$. Note that these values are obtained in CW operation on RAID, so the production of negative ions is in a stationary regime. The data in $\mathrm{H}_{2}$ are up to $8 \mathrm{~kW}$ of power and four gas pressures: $0.2 \mathrm{~Pa}, 0.3 \mathrm{~Pa}, 0.4 \mathrm{~Pa}$ and $0.5 \mathrm{~Pa}$, the data in $\mathrm{D}^{-}$are shown up to $5 \mathrm{~kW}$ at $0.3 \mathrm{~Pa}$ because at the time of the experiments the impedance matching system was not calibrated for powers larger than $5 \mathrm{~kW}$ and we were mostly interested in the pressure relevant for application for NBI, namely 0.3 Pa. We remark that RAID operation at $0.2 \mathrm{~Pa}$ is possible, but results in a reduced performance. For these experimental investigations we chose to operate at $0.3 \mathrm{~Pa}$ as a compromise between a good signalto-noise ratio and the low pressure requirements for the NBI applications. The lineintegrated negative ion density appears to increase with fill pressure, but this is not helpful, given the low pressure requirements for NBI applications.

\subsection{LP-assisted laser photodetachment to determine radial $n_{-}$profiles}

LP-assisted photodetachment has the main advantage of providing information on the local value of negative ion density according to eq. 5 , although it is more perturbing than CRDS. In Fig. 7, typical photodetachment signals in a $\mathrm{H}_{2}$ plasma for increasing RF powers are shown. Photodetachment signals are averaged over 200 laser pulses. To filter out the DC component of the signal collected when the probe bias is at $V_{\text {bias }}$ from the fast transient due to the photodetachment, an AC decoupling circuit, consisting of 


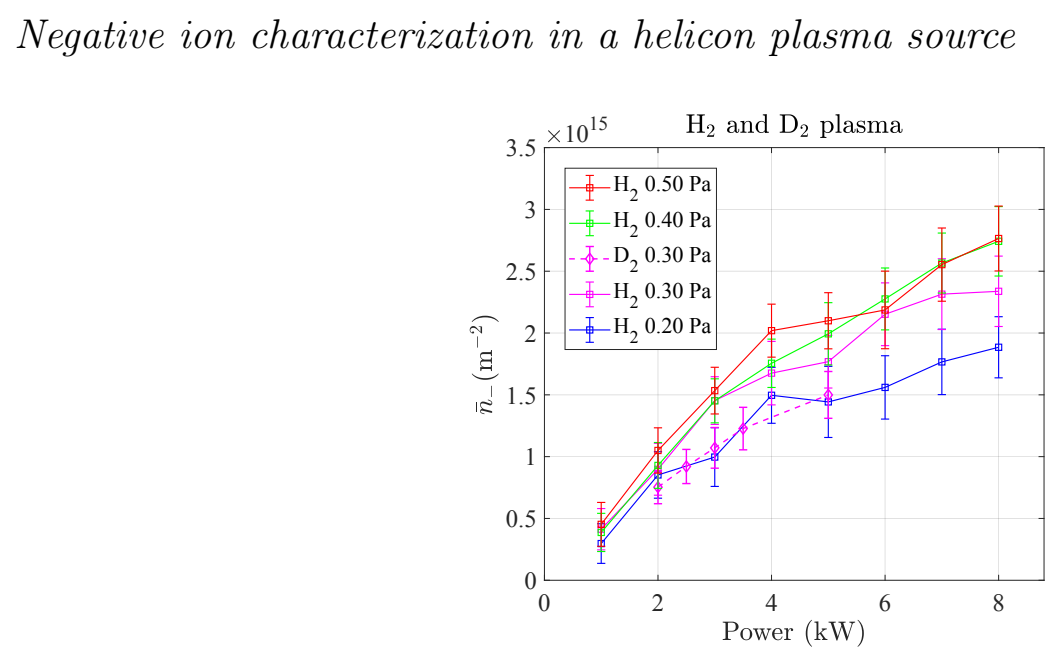

Figure 6. Line-integrated negative ion density as a function of RF power at different pressures for $\mathrm{H}_{2}$ and $\mathrm{D}_{2}$ plasmas, demonstrating the steady-state production of negative ions up to $8 \mathrm{~kW}$ of $\mathrm{RF}$ power.

a high pass filter, was initially used. However, in order to avoid the distortion of the signal due to the bandwidth of the circuit, we finally employed an AC toroidal current transformer (FTC-016, 10:1) with $2.5 \mathrm{~V} / \mathrm{A}$ sensitivity and upper cut-off frequency at $1.17 \mathrm{GHz}$. In Fig. 7, the laser pulse strikes the LP tip at $t=0 \mathrm{~s}$ causing the overshoot (increase of the collected electron current) whose amplitude is indicated by the vertical arrow for the $5 \mathrm{~kW}$ case. The subsequent undershoot and the lack of "plateau" has been reported by other authors such as by P. Devynck et al. [26], M. Bacal [25] and S. Aleiferis [27]. This effect is partly due to the motion of ions caused by the thermal motion of the electrons [28]. The large undershoot and the double peak might also be an effect of the magnetized electrons. Ions are however not magnetized for the magnetic fields employed for these tests (200G). Numerical simulations using a particle-in-cell code are presently under way to fully model the temporal dynamics of the observed photodetachment signal. If the laser pulse strikes the probe when there is no plasma, no variation of the signal is detected. The amplitude increases with RF power confirming the trend observed during CRDS tests.

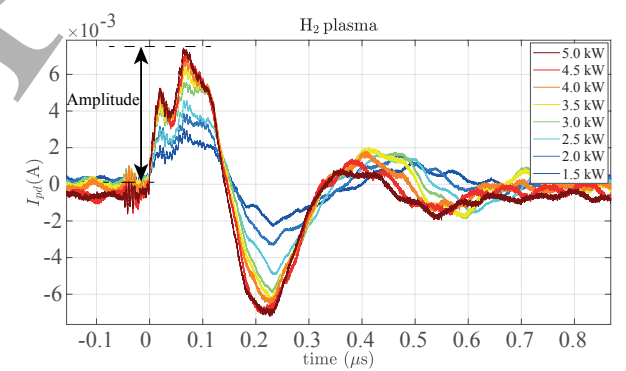

Figure 7. Typical photodetachment signals for a $\mathrm{H}_{2}$ plasma. The laser strikes the LP tip at $t=0 \mathrm{~s}$ causing the overshoot whose amplitude is indicated by the arrow.

We verified that the energy of the laser pulse was enough to strip all the electrons of 


\section{Negative ion characterization in a helicon plasma source}

$\mathrm{H}^{-}$to satisfy (6). This was performed by increasing the laser pulse energy and observing the $I_{p d}$ saturation from a pulse energy of $35 \mathrm{~mJ}$. Consequently, all results were obtained using a pulse energy of $50 \mathrm{~mJ}$.

To radially characterize the plasma, the LP is moved in the vertical direction. For each position, a new alignment laser beam-probe is needed. Fig. 8 summarizes the results obtained with laser photodetachment while varying the RF power. The left and the right column show the results for $\mathrm{H}_{2}$ and $\mathrm{D}_{2}$ plasmas respectively. The gas filling pressure was $0.3 \mathrm{~Pa}$.

In Fig. 8(a) the amplitudes of the photodetachment signals are shown. On average, a weaker signal is measured in the case of $D_{2}$. As suggested by Eq. (6), the shape of the curve is due to a combination of the negative ion density and the electron temperature. Fig. 8(b) shows the electron current $I_{\mathrm{dc}}$ when the probe is biased at $V_{\text {bias }}=26 \mathrm{~V}$, above the plasma potential. On average, more electrons are collected in the case of $\mathrm{D}_{2}$ suggesting that the electron density is higher in this plasma. It was not possible to perform measurements for $r<0.04 \mathrm{~m}$ because $I_{d c}$ was too high and risked damaging the system. Moreover, the window height limited the LOS of the laser to $r<0.065 \mathrm{~m}$.

Fig. 8(c) shows the ratios $n_{\mathrm{H}^{-}} / n_{e}$ and $n_{\mathrm{D}^{-}} / n_{e}$, given by Eq. (6). Although the electrons are magnetized, the electron transport coefficient is the same both for the photodetached electrons and for the electrons collected by the probe when biased above $V_{\text {plasma }}$. Therefore, the transport coefficients tend to cancel out in the ratio. We note the strong difference between the two plasmas. At $r=0.06 \mathrm{~m}, n_{\mathrm{H}^{-}} / n_{e}$ reaches 0.3 , while $n_{\mathrm{D}^{-}} / n_{e}$ reaches about 0.05 . The ratio $n_{\mathrm{H}^{-}} / n_{e}$ is comparable to the value obtained in ICP sources, with moderate source conditioning, close to the extraction grid, at $0.4 \mathrm{~Pa}$ pressure and $55 \mathrm{~kW}$ power, using the same technique [29]. However, a comparable $n_{\mathrm{H}^{-}} / n_{e}$ does not necessarily imply that the extracted negative ion current from a hypothetical helicon source will be comparable to ICP. The magnetic field employed for these experiments (200G) is double compared to the magnetic filter field of ICP sources leading to different transport of the relevant species.

Also, the profile ratios are only weakly dependent on power, as shown in Fig. 8(c). The independence of the ratio $n_{-} / n_{e}$ might be an advantage because it makes the negative ion yield in the source easier to tune. The reason of this difference between $n_{\mathrm{H}^{-}} / n_{e}$ and $n_{\mathrm{D}^{-}} / n_{e}$ is mainly due to the higher electron density measured in $\mathrm{D}_{2}$ plasma, as shown in Fig. 8(d). At $5 \mathrm{~kW}$ power and $r=0.04 \mathrm{~m}$ (where the fixed LOS of CRDS is located) $n_{e}$ attains $1.3 \times 10^{17} \mathrm{~m}^{-3}$ in $\mathrm{H}_{2}$ and $2.9 \times 10^{17} \mathrm{~m}^{-3}$ in $\mathrm{D}_{2}$. Although $n_{e}$ might be unreliable because of electron magnetization, this data completes the set of quantities which can be obtained only relying on the LP laser photodetachment technique.

The $n_{e}$ profiles are obtained from the analysis of IV curves taken with the same LP used for laser photodetachment. The electron energy distribution function (EEDF) is calculated using the Druyvesteyn formula [30] and the second derivative of the measured electron current is calculated with a Savitzky-Golay filter to obtain the floating and plasma potential. An effective electron temperature $T_{e}$ and the total electron density are then obtained by numerically integrating the EEDF. More details on the analysis 
Negative ion characterization in a helicon plasma source

(a)
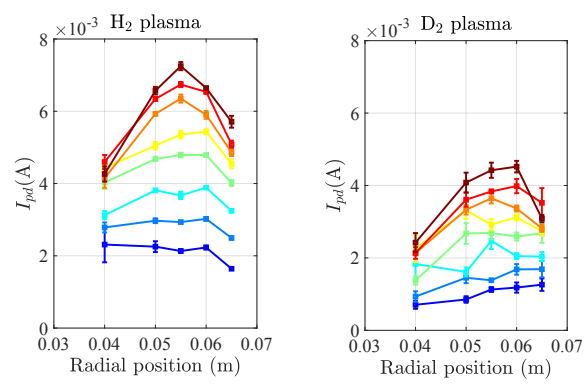

(b)
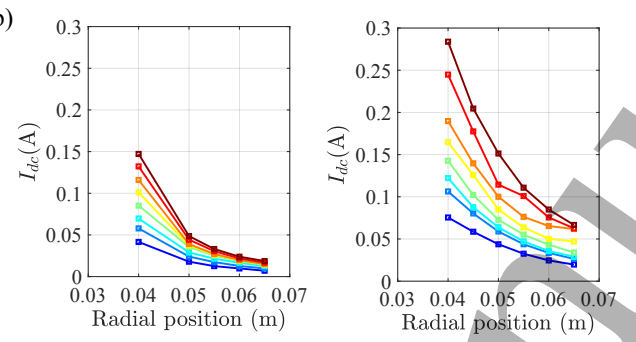

(c)

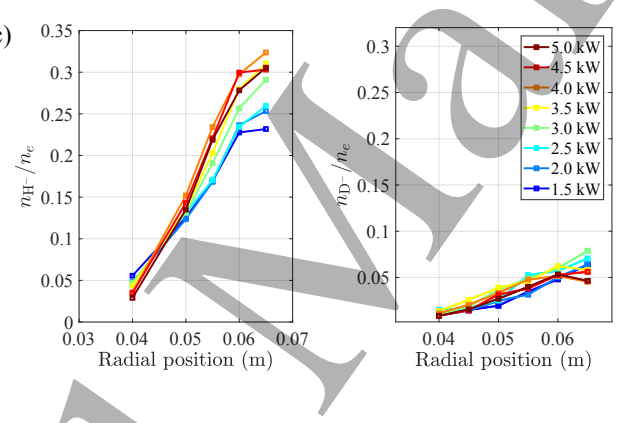

(d)
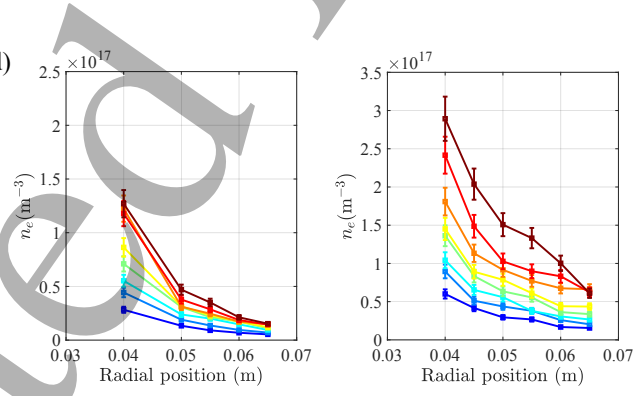

Figure 8. Results of the laser photodetachment measurements for $\mathrm{H}_{2}$ (left column) and $\mathrm{D}_{2}$ (right column) plasmas at $0.3 \mathrm{~Pa}$ : (a) Photodetachment amplitudes $I_{p d}$; (b) Electron current $I_{d c}$ collected when the probe is biased at $V_{\text {bias }}=26 \mathrm{~V}$, above the plasma potential; (c) Ratio $n_{-} / n_{e}$; and (d) Electron density $n_{e}$. On the edge of the plasma column, hydrogen plasma has lower electron density and a higher ratio $n_{-} / n_{e}$.

can be found in [31]. The higher density in $\mathrm{D}_{2}$ is measured by microwave interferometry at the edge, at measurement position $r=0.04 \mathrm{~m}$, revealing a line-integrated density a factor 2 higher in the case of deuterium.

The data shown in Fig. 8(a), (b) and (d) can be used to calculate the negative ion density, which is reported in Fig. 9. Negative ion density peaks at $r \sim 0.055 \mathrm{~m}$ and $\mathrm{H}^{-}$and $\mathrm{D}^{-}$ are distributed in a shell-like shape, as also measured by OES [6]. The peak density of $\mathrm{H}^{-}$is $7.7 \times 10^{15} \mathrm{~m}^{-3}$ which is a factor $\sim 2.5$ lower compared to $2.0 \times 10^{16} \mathrm{~m}^{-3}$ measured 
via OES [6]. The disagreement between OES and laser photodetachment might be due to an imprecise estimate of $n_{e}$ via LPs.
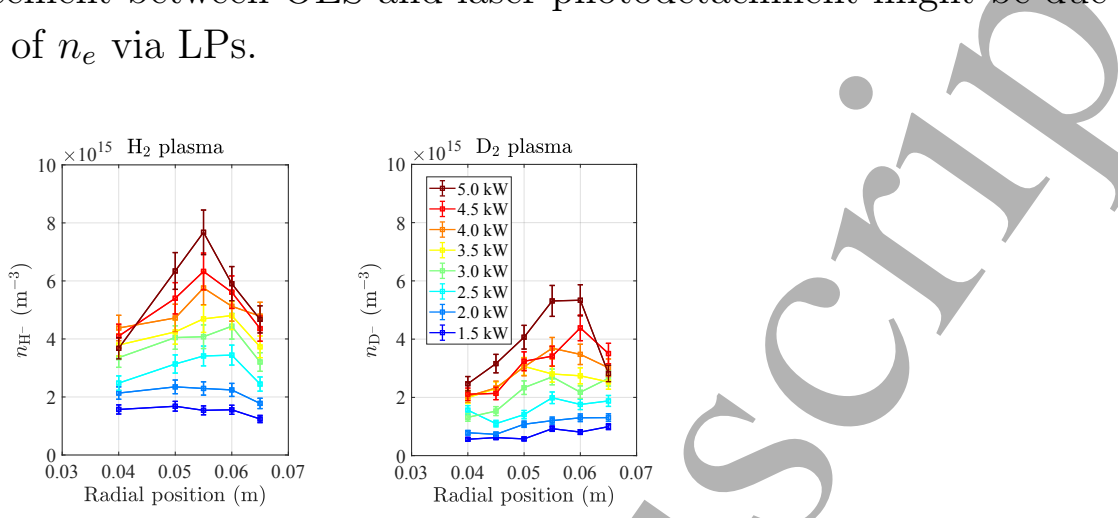

Figure 9. Radial profiles of negative ion density obtained with laser photodetachment and measuring the electron density via IV curves. These data are not calibrated with CRDS.

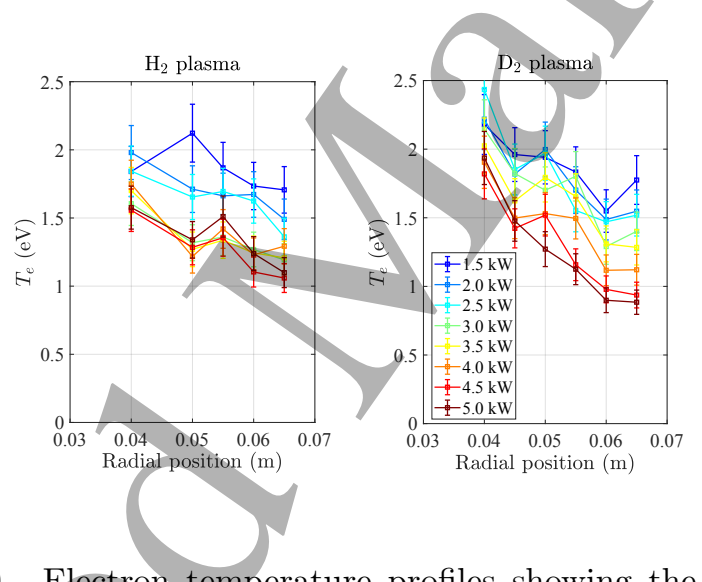

Figure 10. Electron temperature profiles showing the weak dependence with the $\mathrm{RF}$ power and the weak variation over the range of measurement. The low value of electron temperature in this range is favorable for the production of negative ions by dissociative attachment (DA).

For completeness we also show the temperature profiles in Fig. 10. $T_{e}$ decreases weakly along the radial direction down to $1 \mathrm{eV}$ at $r=0.065 \mathrm{~m}$ and $5 \mathrm{~kW}$ of power for both $\mathrm{H}_{2}$ and $\mathrm{D}_{2}$. Moreover, a general decrease of $T_{e}$ is observed for increasing $\mathrm{RF}$ power. The $T_{e}$ values in the edge are in the range where the DA cross section peaks, depending on the rovibrational state of $\mathrm{H}_{2}(v)$ and $\mathrm{D}_{2}(v)$. Thus, the $T_{e}$ profiles show that the electron cooling on the edge, caused by the magnetic field needed to sustain the helicon discharge, is also effective to reach a favorable electron temperature for DA process.

4.3. CRDS combining with LP-assisted laser photodetachment to extract absolute negative ion density profiles

In this section, we focus only on the data at $0.3 \mathrm{~Pa}$, since this is the pressure relevant to present NBIs systems, such as the system designed for ITER, although lower pressure 


\section{Negative ion characterization in a helicon plasma source}

may be more relevant for future systems [32]. The measurement of $n_{e}$ via LPs in a plasma where electron are magnetized, might lead to error on the final estimate of negative ion density, $n_{-}$, when $n_{-}$is deduced from (6). To overcome this problem one can use the line-integrated negative ion density measured with CRDS for absolute calibration. The negative ion density profiles obtained with laser photodetachment shown in Fig.9(a) and Fig. 9(b) might be not precise in absolute value but anyway proportional to $n_{-}$. Thus, they can be still used to extract the width and the position of the negative ion shell. Fig. 11(a) and (b) show the relative $n_{-}$profiles with the Gaussian fits, the position of CRDS line-of-sight and of the vessel wall.
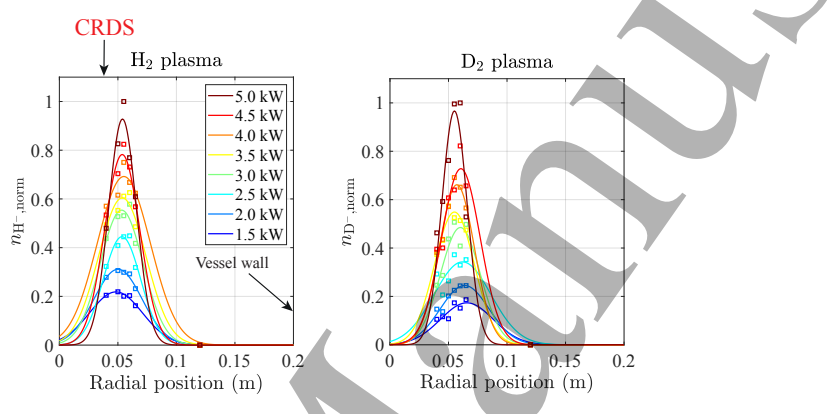

Figure 11. Gaussian fits of the relative $n_{-}$profiles measured with laser photodetachment. The position of CRDS line-of-sight to calibrate $n_{-}$absolute profiles and the vessel wall position is indicated.

The fit is performed with a Gaussian function, based on previous $n_{\mathrm{H}^{-}}$profiles measured with OES [6]. The region at $r<0.04 \mathrm{~m}$ is not taken into account for the estimate of the absolute $n_{-}$profile since CRDS measures the density outside this measurement position. We define $F(x, y)$ as the normalized $n_{-}$profile at a certain RF power, where $x$ and $y$ are coordinates in the plane transverse to the plasma column axis, shown in Fig. 4(b). $F(x, y)$ is the function having the role of form factor, thus giving the shape of the negative ion density profile. The absolute negative ion density $n_{-}(x, y)$, shown in Fig. 4(b), can be written as:

$$
n_{-}(x, y)=n_{-}^{\text {peak }} F(x, y)
$$

where $n_{-}^{\text {peak }}$ is the negative ion peak density. CRDS measures the integral of (7) along the line of sight at the measurement position $y=y_{C R D S}$, which is:

$$
C_{-}^{R D S}=n_{-}^{\text {peak }} \int_{x_{1}}^{x_{2}} F\left(x, y_{C R D S}\right) d x
$$

where $x_{1}$ and $x_{2}$ are the integration limits along the line of sight. From (8) one can deduce $n_{-}^{\text {peak }}$ which, inserted in (7), provides the absolute negative ion density profile. Fig. 12 shows the absolute $n_{\mathrm{H}^{-}}$and $n_{\mathrm{D}^{-}}$profiles only for 2,3 and $5 \mathrm{~kW} \mathrm{RF}$ powers for clarity. The peak value obtained at $5 \mathrm{~kW}$ for $\mathrm{H}_{2}$ is $1.9( \pm 0.3) \times 10^{16} \mathrm{~m}^{-3}$, close to the value measured by OES [6], which was $3.0( \pm 0.8) \times 10^{16} \mathrm{~m}^{-3}$. For $\mathrm{D}^{-}$the peak value 
1

2

3

4

5

6

7

8
Negative ion characterization in a helicon plasma source
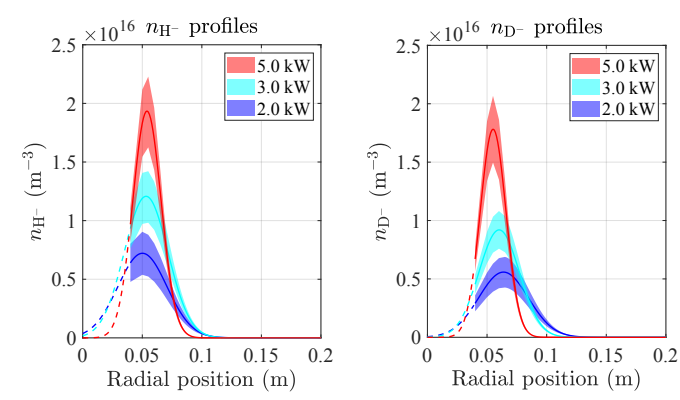

Figure 12. $n_{\mathrm{H}^{-}}$and $n_{\mathrm{D}^{-}}$radial profiles for different powers obtained using the relative negative ion profiles measured with laser photodetachment and calibrated with CRDS line-integrated measurements.

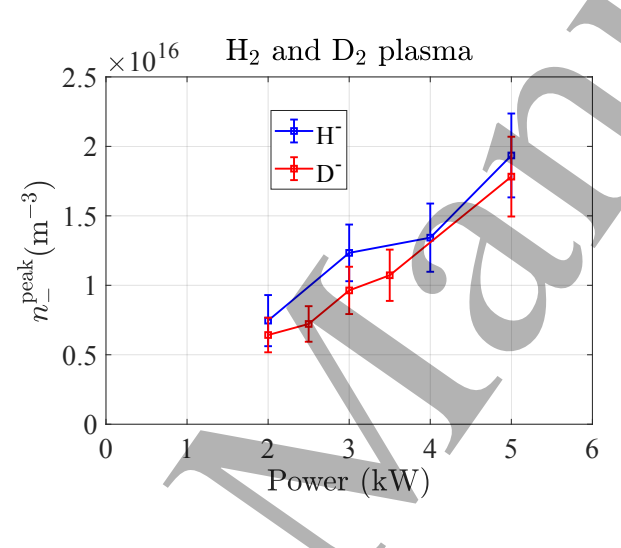

Figure 13. Peak negative ion density as a function of RF power at $0.3 \mathrm{~Pa}$. $n_{-}$ increases with power and higher density is observed in the case of $\mathrm{H}^{-}$.

for $5 \mathrm{~kW}$ results in $1.8( \pm 0.3) \times 10^{16} \mathrm{~m}^{-3}$, a bit low compared to $4.5( \pm 2.0) \times 10^{16} \mathrm{~m}^{-3}$ measured with OES.

In Fig. 13, we plot the peak value of $n_{-}$for increasing powers, showing a linear trend. The isotope effect seems to result in a slightly higher negative ion density for the case of hydrogen, but this is comparable to the error bar. Furthermore, in Fig. 14 we plot the negative ion density with electron density measured by the LP at $r=0.055 \mathrm{~m}$, close to $n_{-}$peak yalues. $n_{-}$increases almost linearly with $n_{e}$ and higher values may be attained by increasing the $\mathrm{RF}$ power. Even though the absolute value of $n_{e}$ might be incorrect, the lower ratio $n_{-} / n_{e}$ in the case of deuterium is clearly visible. This suggests that the co-extracted electrons might be a more important issue for $\mathrm{D}^{-}$extraction.

The isotopic effect in negative ion and electron density has been investigated by some authors and it is due to a combination of different phenomena [33, 34, 35]. Calculations and experiments show that $n_{e}$ is higher in deuterium than hydrogen plasmas and is mostly due to ion transport. Positive ions are indeed mostly lost by transport to the walls where they are neutralized. For deuterium, the transport of positive ions is a factor $1 / \sqrt{2}$ smaller than hydrogen because of the mass difference. This might explain in part the higher $n_{e}$ in deuterium [33]. Concerning the isotope 


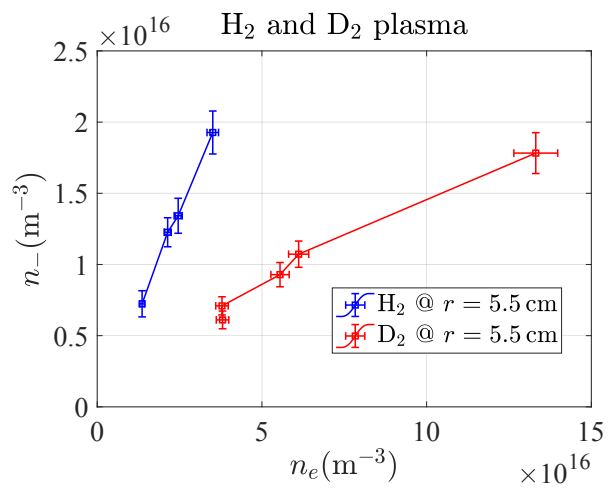

Figure 14. Negative ion density vs. electron density in hydrogen and deuterium plasmas.

effect on the negative ion density, the lower negative ion density in deuterium might be due to the higher degree of dissociation in deuterium leading to increased V-t cooling (cooling of $\mathrm{D}_{2}(\mathrm{v})$ in collision with $\mathrm{D}$ ) and then less efficient $\mathrm{D}^{-}$production [33]. For a better understanding of the processes, modeling is envisaged.

\section{Discussion and Conclusions}

The measurements performed with CRDS and LP-assisted photodetachment show that $\mathrm{H}^{-}$and $\mathrm{D}^{-}$are produced both in hydrogen and deuterium helicon plasmas in steady state regime with significant densities in volume production mode, without using caesium and with a few $\mathrm{kWs} R F$ power. The measurements were performed at a fill pressure of $0.3 \mathrm{~Pa}$, relevant to NBIs of ITER. Future NBIs may require lower fill pressures, and future studies at those pressures will be required [32]. We showed that LPassisted photodetachment is not sufficient alone to obtain reliable measurements. Thus, to extract the absolute negative ion density profile, LP-assisted laser photodetachment is employed in combination with line-integrated measurements of CRDS. The LP-assisted laser photodetachment is used to measure the relative negative ion density profile showing that it is peaked off-axis. The line-integrated measurement performed by CRDS is then used to obtain the absolute negative ion density profile. Taking into account the peak negative ion density at $5 \mathrm{~kW} R F$ power, $\mathrm{H}^{-}$density reaches $1.9( \pm 0.3) \times 10^{16} \mathrm{~m}^{-3}$, close to $3.0( \pm 0.3) \times 10^{16} \mathrm{~m}^{-3}$ measured with OES. However, $\mathrm{D}^{-}$reaches $1.8 \times 10^{16} \mathrm{~m}^{-3}$ quite lower if compared to $4.5( \pm 2.0) \times 10^{16} \mathrm{~m}^{-3}$ measured with OES. The reason for this disagreement in the $\mathrm{D}_{2}$ case is under investigation. Despite the absolute $\mathrm{H}^{-}$and $\mathrm{D}^{-}$ densities appear to be comparable, the ratio $n_{-} / n_{e}$ is about 6 times lower in deuterium than hydrogen. This suggests that the co-extracted electrons might be a more important issue foy deuterium, if the current magnetic geometry is employed.

We believe that these experimental investigations add relevant data on the volume production of $\mathrm{H}^{-}$and $\mathrm{D}^{-}$in hydrogen and deuterium helicon plasmas. However, the design of a source based on a helicon plasma requires the investigation of different 


\section{Negative ion characterization in a helicon plasma source}

magnetic field geometries and the compatibility with an extractor and an acceleration system. Thus, a next step with the current system is the characterization of negative ions along the plasma column and the study of different magnetic geometries, such as the expanding magnetic field, with the diagnostics mentioned in this paper. In parallel with this, 3D numerical simulations could be an important tool to predict the negative ion production as a function of source parameters.

If used as part of a future ion source for an NBI system, direct extraction of the negative ions from the plasma column in the radial or axial direction should be possible. If radial extraction is used, because of the inherent cylindrical nature of the helicon driven plasma, the beam would have to be long and narrow, such as that suggested in reference [36]. However significant development may be needed if the extractor perturbs significantly the plasma and because the extraction system would need to be segmented, with each segment fitting between the magnetic coils. Axial extraction seems unattractive as the annular region from which ions could be extracted is short, and hence the extracted current would be low, and a transverse filter field would probably be needed to reduce the co-extracted electron current. It is also to be noted that the resulting annular beam shape has no particular advantage from the point of view of neutralisation or beamline design.

Using the helicon device to provide a plasma that expands towards a conventional multi-aperture system with a caesiated plasma grid should be considered because of the ease of operation at fill pressures $<0.3 \mathrm{~Pa}$ and the high efficiency of the helicon device. In this case, it needs to be established that the helicon driven plasma produces sufficient dissociation of the gas in order to have sufficient negative ion production via the impingement of the atoms on the low work function of the plasma grid surface.

\section{Acknowledgments}

This work has been carried out within the framework of the EUROfusion Consortium and has received funding from the Euratom research and training programme 2014 - 2018 and 2019 - 2020 under grant agreement No 633053. The views and opinions expressed herein do not necessarily reflect those of the European Commission. This work was supported in part by the Swiss National Science Foundation. We gratefully acknowledge the mechanical and electronic workshops of the Swiss Plasma Center for their collaboration.

\section{Bibliography}

[1] G. Serianni, P. Agostinetti, M. Agostini, D. Aprile, C. Baltador, M. Barbisan, M. Brombin, M. Cavenago, G. Chitarin, M. Dalla Palma, R. Delogu, F. Fellin, N. Fonnesu, N. Marconato, R. Pasqualotto, A. Pimazzoni, E. Sartori, S. Spagnolo, M. Spolaore, P. Veltri, B. Zaniol and M. Zaupa, New J. Phys. 19, 045003 (2017). 
Negative ion characterization in a helicon plasma source

[2] R. S. Hemsworth, D. Boilson, P. Blatchford, M. Dalla Palma, G. Chitarin, H.P.L. de Esch, F. Geli, M. Dremel, J. Graceffa, D. Marcuzzi, G. Serianni, D. Shah, M. Singh, M. Urbani and P Zaccaria, New J. Phys. 19, 025005 (2017).

[3] U. Fantz, C. Hopf, D. Wünderlich, R. Friedl, M. Fröschle, B. Heinemann, W. Kraus, U. Kurutz, R. Nocentini and L. Schiesko, Nucl. Fusion 57, 116007 (2017).

[4] P. Sonato, P. Agostinetti, U. Fantz, I. Furno, A. Simonin and M. Q. Tran, New J.Phys. 18, 125002 (2016).

[5] S. Briefi and U. Fantz, AIP Conf. Proc., 1515, 278 (2013).

[6] C. Marini, R. Agnello, B. P. Duval, I. Furno, A. A. Howling, R. Jacquier, A. N. Karpushov, G. Plyushchev, K. Verhaegh, and Ph. Guittienne, Nucl. Fusion 57, 036024 (2017).

[7] S. Shinoara, Advances in Physics: X, 3:1, 3(1):1420424, (2018).

[8] I. Furno, R. Agnello. U. Fantz, A. Howling, R. Jacquier, C. Marini, G. Plyushchev, Ph. Guittienne, and A. Simonin, EPJ Web Conf. 157, 03014 (2017).

[9] J. Santoso, R. Manoharan, S. O’Byrne and C. S. Corr, Phys. Plasmas 22, 093513 (2015).

[10] A. Pandey, D. Mukherjee, D. Borah, M. Bandyopadhyay, H. Tyagi, R. Yadav and A. Chakraborty Plasma Phys. Control. Fusion 61, 065003 (2019).

[11] P. Guittienne, E. Chevalier and Ch. Hollenstein, J. Appl. Phys. 98, 083304 (2005).

[12] I. Furno, R. Agnello, A. A. Howling, R. Jacquier, G. Plyushchev, Ph. Guittienne, M. Barbisan, R. Pasqualotto, S. Béchu, I. Morgal and A. Simonin, 60th Annual Meeting of the APS Division of Plasma Physics, Portland, Oregon (USA).

[13] R. Jacquier, R. Agnello, B. Duitel, C. Marini, Ph. Guittienne, A. A. Howling, I. Furno, G. Plyushchev, A. Simonin, I. Morgal and S. Béchu, Fusion Eng. Des. 146, 1140-1144 (2019).

[14] R. Agnello, M. Barbisan, I. Furno, Ph. Guittienne, A. Howling, R. Jacquier, R. Pasqualotto, G. Plyushchev, Y. Andrebe, S. Béchu, I. Morgal and A. Simonin, Rev. Sci. Instrum. 89, 103504 (2018).

[15] M. Berger, U. Fantz, S. Christ-Koch and NNBI, Team, Plasma Sources Sci. Tech. 18, 025004 (2009).

[16] M. A. Lieberman and A. J. Lichtenberg, Principles of plasma discharges and materials processing (John Wiley and Sons, New York, 1994).

[17] I. D. Sudit and F. F. Chen, Plasma Sources Sci. Technol. 5, 43 (1996).

[18] J. N. Bardsley, J. Phys. B: At. Mol. Phys. 1, 349 (1968).

[19] J. S. Schultz, Rev. Mod. Phys. 45, 423 (1973).

[20] M. Bacal, Nucl. Fusion 46, S250-S259 (2006).

[21] J. R. Hiskes, J. Appl. Phys. 51, 4592 (1980).

[22] A. O'Keefe and D. G. Deacon, Rev. Sci. Instrum. 59, 2455 (1988).

[23] H. Nakano, K. Tsumori, M. Shibuya, S. Geng. M. Kisaki, K. Ikeda, K. Nagaoka, M. Osakabe, Y. Takeiri and O.Kaneko, in 17th International Symposium on Laser-Aided Plasma Diagnostics, (IOP Publishing, 2015), p.3.

[24] R. Pasqualotto, A. Alfier and L. Lotto, Rev. Sci. Instrum. 81, $10 \mathrm{D} 710$ (2010).

[25] M. Bacal, Rev. Sci. Instrum. 71, 3981 (2000).

[26] P. Devynck, J. Auvray, M. Bacal, P. Berlemont, J. Bruneteau, R. Leroy, and R. A. Stern, Rev. Sci. Instrum. 60, 2873 (1989).

[27] S. Aleiferis, Experimental Study of $\mathrm{H}^{-}$negative ion production by electron cyclotron resonance plasmas, Université Grenoble-Alpes, $\mathrm{PhD}$ thesis (2016).

[28] L. Friedland, C. I. Ciubotariu, and M. Bacal, Phys. Rev. E 49, 4353 (1994).

[29] S. Christ-Koch, U. Fantz, M. Berger and NNBI Team, Plasma Sources Sci. Tech. 18, 025003 (2009).

[30] M. J. Druyvesteyn, Z. Phys. 64, 781 (1930).

[31] S. Béchu, A. Soum-Glaude, A. Bs, A. Lacoste, P. Svarnas, S. Aleiferis, A. A. Ivanov and M. Bacal Phys. Plasmas 20, 101601 (2013).

[32] R. S. Hemsworth and D. Boilson, AIP Conference Procedings 1869, 060001 (2017). 
[33] D. A. Skinner, A. M. Bruneteau, P. Berlemont, C. Courteille, R. Leroy, and M. Bacal, Phys. Rev. E 48, 2122 (1993).

[34] A. M. Bruneteau, C. Courteille, R. Leroy, and M. Bacal, Rev. Sci. Instrum. 67, 3827 (1996).

[35] O. Fukumasa and K. Yoshino, Rev. Sci. Instrum. 69, 941 (1998).

[36] A. Simonin, R. Agnello, S. Béchu, J. M. Bernard, C. Blondel, J. P. Boeuf, D. Bresteau, G. Cartry, W. Chaibi, C. Drag, B. P. Duval, H. P. L. de Esch, G. Fubiani, I. Furno, C. Grand, Ph. Guittienne, A. Howling, R. Jacquier, C. Marini and I. Morgal, New J. Phys. 18, 125005 (2016). 